This is a self-archived version of an original article. This version may differ from the original in pagination and typographic details.

Author(s): Mulat Gebrekirstos, Mekonnen; Savolainen, Hannu; Lehtomäki, Elina; Kuorelahti,

Title: Socio-emotional Problems Experienced by Deaf and Hard of Hearing Students in Ethiopia

Year: 2015

Version: Accepted version (Final draft)

Copyright: (c) 2015 Taylor \& Francis

Rights: In Copyright

Rights url: http://rightsstatements.org/page//nC/1.0/?language=en

Please cite the original version:

Mulat Gebrekirstos, M., Savolainen, H., Lehtomäki, E., \& Kuorelahti, M. (2015). Socio-emotional Problems Experienced by Deaf and Hard of Hearing Students in Ethiopia. Deafness and Education International, 17(3), 155-162. https://doi.org/10.1179/1557069X15Y.0000000002 


\section{Socio-emotional Problems Experienced by Deaf and Hard of Hearing Students in Ethiopia}

Original research paper

Deafness \& Education International Journal

Published online on 4 Feb 2015 at http://dx.doi.org/10.1179/1557069X15Y.0000000002

There are no conflicts of interests. The schools involved in this study have received informed consents from the research participants, parents or guardians.

\section{Acknowledgement}

We are grateful to all students participated in this study for their cooperation and to their teachers who helped us in the practical arrangements when carrying out this study.

\section{Authors:}

Mulat Mekonnen (corresponding author), mekonnen.mulat@nic.fi

Savolainen Hannu, University of Jyväskylä Finland, hannu.savolainen@jyu.fi

Lehtomäki Elina, University of Jyväskylä, Finland, elina.k.lehtomaki@jyu.fi

Kuorelahti Matti, University of Jyväskylä Finland, matti.kuorelahti@jyu.fi

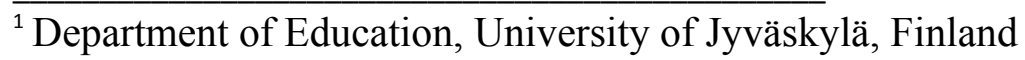




\section{Abstract}

This study compares the socio-emotional problems experienced by deaf and hard of hearing (DHH) students with those of hearing students in Ethiopia. The research involved a sample of 103 gradefour students attending a special school for the deaf, a special class for the deaf and a regular school. Socio-emotional problems were measured using Goodman's self-report measure, the Strengths and Difficulties Questionnaire (SDQ) for children and adolescents. Participants were selected from seven towns in Ethiopia. The results show that, compared to the hearing sample, the DHH students experienced more severe socio-emotional problems across all dimensions, regardless of whether they were in special classes or special schools. The DHH children reported that socioemotional difficulties interfered with their home lives, friendships, classroom learning and ability to get along with the people around them. The DHH students scored higher in the externalizing behavioural domains than did the hearing students. There was no statistically significant difference between the DHH students in the special school and special class settings. The results show that DHH children expressed great concern about their socio-emotional difficulties. Teachers and other professionals need to raise their awareness about DHH issues in order to help these children overcome their challenges.

KEYWORDS: Deafness; Hearing impairment; Deaf students; Hard-of-hearing students; Socioemotional problems; Deaf education; Ethiopia, Africa 


\section{Introduction}

Previous studies on cognitive development and functioning show that deaf and hard of hearing (DHH) students face special challenges. Studies show that DHH children are more impulsive than hearing children due to the developmental delay resulting from early language deprivation (Marschark \& Wauters, 2011). Research into socio-emotional development (Calderon \& Greenberg, 2011) reveals that reduced auditory perception influences many processes that are significant for children's effective interaction with the world. The prevalence of mental health problems in DHH students remains difficult to establish for a number of reasons such as the heterogeneous nature of students with hearing loss relating to the degree and type of hearing loss, age of onset, time of diagnosis, communication and cultural identification. Other significant issues are the appropriateness of instruments used and the informants who provide the data (Brown \& Cornes, 2014). Therefore, attention must be given to socio-emotional development when raising and educating DHH children. The present study investigates the impact of socio-emotional problems in the lives of DHH children attending special schools and special classes compared to hearing students in Ethiopia. It also examines whether there are differences in the self-reported externalizing behaviour patterns between the three groups (students in special school, in special class, and hearing students). To our knowledge, the socio-emotional development of DHH children in Africa has yet to be studied.

\section{Educational settings for DHH students in Ethiopia}

According to recent survey data reported by the World Health Organization (WHO, 2013), 360 million people worldwide have disabling hearing loss, and two-thirds of them live in developing countries. In most developing countries, the incidence and causes of hearing impairment remain largely undocumented due to limited resources and access to health care as well as lack of screening 
and early intervention services. In sub-Saharan Africa, these limitations combined with lack of information and traditional beliefs and practices, cause preventable hearing losses (Olusanya, 2008). In Ethiopia, there is no reliable data indicating the total number of DHH people or the number of school-aged DHH children. The national average gross enrolment rate at the primary level for all types of children with disabilities was approximately 3.2\% (MoE, 2012), implying that 96.8\% of children with disabilities remain unserved by the education system, often remaining out of school. For the minority of DHH children who have accessed education, placement options can be categorized into three broad types: (a) schools exclusively for DHH students, which includes day/residential schools; (b) special classes within the regular public schools, allowing DHH and hearing students social interaction during their free time and extracurricular activities; and (c) regular public schools, typically with limited DHH peers integrated with hearing students (often referred to as inclusive). The special classes provide education for DHH children up to grade four before integrating them with regular hearing students beginning in grade five. In 2013, there were 13 special schools and 302 special classes. Traditionally, DHH students have been placed primarily in schools exclusively for DHH students. In recent years, placement has shifted considerably, and the global trend has been to educate DHH children in inclusive settings (Marschark \& Knoors, 2012), which is likely to be the case for Ethiopia in the future.

In Ethiopia, however, the challenge is getting DHH children to any form of schools. To address this issue, the Ministry of Education (MoE), with support from Finland, designed the first SNE Program Strategy in 2006 (Ayana \& Lehtomäki, 2006). The strategy focuses on the promotion of inclusive education to meet the Millennium Development Goals and the Education for All Goals. In 2012, the strategy was revised and re-released in April 2013 along with implementation guidelines (MoE, 2012). The government also has referred to international conventions, declarations and statements related to inclusive education after ratifying the UN Convention on the Rights of the Child in 1991 and the UN Convention on the Rights of Persons with Disabilities in 
2010. The Ethiopian Constitution (Article 9) affirms that all international agreements ratified by Ethiopia are an integral part of the law. Within this legal framework, the government, along with other stakeholders, is moving forward in addressing the educational needs of DHH children.

Nonetheless, DHH learners face several major challenges in Ethiopian regular classrooms, including the inaccessibility of sign language. The size of the regular class may be very large (60 80 students per class), and most of the regular class teachers do not know sign language or understand the communicational needs of $\mathrm{DHH}$ children. There are no educational sign language interpreters in the country, except in the capital city, Addis Ababa. This situation has created a challenge for most deaf students in continuing their schooling. Due to lack of appropriate support, more specifically support in sign language and communication, deaf children (especially those who are pre-lingual) find learning very difficult in an environment that demands hearing; therefore, many of them are forced to drop out of school (Mulat, 2011).

\section{Behavioural and emotional problems in DHH children}

Behavioural and emotional disorders include a wide range of behavioural patterns, ranging from externalizing to internalizing behaviour disorders as well as combined behaviours that include features of both subtypes. Externalizing behaviours are actions that direct problematic energy outward and may include behaviours such as verbal and physical aggression, hyperactivity, noncompliance, and delinquent acts. In contrast, internalizing behaviours are directed toward the self that include social withdrawal, depression, substance abuse, feeding and eating disorders, anxiety, and schizophrenia (for a review, see Kauffman \& Landrum, 2013). Most current studies on DHH students show that, regardless of educational placement, they have significantly higher rates of behavioural and emotional difficulties than hearing students. Various studies have compared the total scores for problems between DHH students and hearing students using the Strengths and Difficulties Questionnaire (SDQ; Goodman, 1997) or the Child Behaviour Checklist (Achenbach, 
1991). These studies are in agreement that DHH students show up to a 2.6-fold higher rate of disorders than their hearing counterparts (Dammeyer, 2010; Fellinger et al., 2008; Hintermair, 2007; van Eldik et al., 2004; van Gent et al., 2007). Dammeyer (2010) reported that the prevalence of psychosocial difficulties in DHH children in Denmark was 3.7 times greater compared to a group of hearing children; DHH students who had additional handicaps were particularly affected. However, as Cornes and Brown (2014) noted, it is challenging to diagnose emotional disturbance in deaf children because the instruments available are difficult for deaf children to understand. In an effort to overcome this, studies made in Australia by Cornes et al. (2006) have used a signed version of the Youth Self Report and found higher prevalence of psychological problems among deaf adolescents than that reported for the wider Australian adolescent population.

\section{Educational settings and psychosocial difficulties in DHH children}

There is no overall agreement on how educational placement of DHH students into mainstream classes, regular school settings or more specialized arrangements affects their psychosocial difficulties. Hintermair (2013) and Keilmann, Limberger and Mann (2007) found low levels of psychosocial difficulties among DHH children attending mainstream schools compared to those in special schools. Earlier researchers, such as Hindley, Hill, McGuigan and Kitson (1994), found the level of psychosocial difficulties lower at special schools compared to DHH units. Polat (2003) documented better psychosocial adjustment patterns in residential school students compared to mainstream school students. Generally, additional disabilities (physiological and/or psychiatric disorders) have been associated with a higher prevalence of psychosocial difficulties among the deaf (van Eldik et al., 2004; van Gent et al., 2007; Hindley et al., 1994; Hintermair, 2007; Polat, 2003; Sinkkonen, 1994; Dammeyer, 2010). As in studies concerning hearing children, parental resources and stress socioeconomic status and ethnicity also correspond to psychosocial wellbeing among DHH children (Stacey et al., 2006). 
The globally well-known controversy between the medical/audiological and cultural/ecological perspectives on deafness has emerged in discussions regarding the psychological development of DHH children with and without cochlear implants (Hindley et al., 1994; Polat, 2003). A bicultural (deaf and hearing) and bilingual (signed and oral) perspective has proven to be positive for deaf children's psychosocial and cognitive development because it gives the children a greater ability for natural communication from early in life; it also provides the opportunity to develop a positive self-image and self-esteem as a deaf person (Preisler, 1999). The research in deaf identity carried out by Bat-Chava (2006) also indicated that deaf people with bicultural identities would have higher self-esteem. Adequate communication is crucial for deaf children's development, and sign language abilities correlate with psychosocial well-being (Polat, 2003; Sinkkonen, 1994). Parental hearing status also has been discussed as an important issue (Mayberry, 2003). Deaf children of deaf parents have shown better psychosocial adjustment than deaf children of hearing parents; as a result, discussions are ongoing about whether it is deafness itself or communication factors that influence the psychosocial well-being of children with hearing loss (Polat, 2003).

Recent studies show that, regardless of the mode of communication and language chosen, DHH children lag behind their hearing peers in cognitive development and academic performance (Marschark \& Knoors, 2012). Almost all of the research available and reviewed has focused on DHH children in high-income countries, countries with conditions for early detection and intervention as well as rehabilitation and supportive measures in schools. Therefore, it is important to investigate the effects of educational placement on the behavioural and emotional wellbeing and academic performance of DHH children in low-income countries with limited resources and services, such as Ethiopia.

Using a sample of Ethiopian students, this study aimed to examine whether the impacts of socio-emotional problems differ among DHH and hearing primary school students in three different 
educational settings (students in special school, students in special class, and hearing students). The study also investigates whether there are differences in the self-reported externalizing behaviour problems between DHH students in special classes, special schools and hearing students. This research will help professionals and educators globally to better understand the problems related to the socio-emotional aspects of DHH children and to make appropriate interventions promoting the socio-emotional development of these children. On one hand, the study has international significance reflecting the African perspective of $\mathrm{DHH}$ education; for the wider audience, it compares the impacts of socio-emotional problems of DHH students in different educational settings.

\section{Methods}

\section{Participants}

The present study included a total of 103 fourth grade students who were attending three types of schools: DHH students in special classes attached to regular schools, DHH students in special schools and hearing students in regular schools. The characteristics of the students who participated in this study are described in Table 1. They were from the Southern Nations, Nationalities and Peoples' Region (from Hossana, Arbminch and Hawassa Town Administrations), Oromia Region (from Asella and Adama Town Administrations), Amhara Region (from Bahir Dar Town Administration) and from Addis Ababa City Administration. The participants were selected purposefully due to the small number of fourth-grade DHH students in special classes. Among the participants, 29 were DHH students from special classes (mean age $=15.4$, SD $=2.9$ ), 31 were DHH students from special schools (mean age $=13.1, \mathrm{SD}=1.7$ ) and 43 were hearing students from regular schools (mean age $=12.1, \mathrm{SD}=1.7$ ) where special classes were attached (Table 1$)$. The age of DHH participants in special classes ranged from 10 to 22 years old. Almost all the students in the 
special classes and special schools had severe to profound bilateral hearing loss, except one student from each setting who was hard of hearing. The researcher measured the hearing levels of DHH students using the pure tone audiometer.

Table 1 Participants of the study $(\mathrm{N}=103)$

\begin{tabular}{lccccccc} 
& & Age & \multicolumn{2}{c}{ Hearing level } & \multicolumn{2}{c}{ Gender } \\
School setting & $\mathbf{N}$ & Mean (SD) & $\mathbf{2 7 0 d B}$ & $>\mathbf{7 0 d B}$ & Male & Female & $\%$ \\
DHH in special class & 29 & $15.4(2.9)$ & 1 & 28 & 13 & 16 & $28.2 \%$ \\
DHH in special school & 31 & $13.1(1.7)$ & 1 & 30 & 16 & 15 & $30.1 \%$ \\
Hearing in regular school & 43 & $12.1(1.7)$ & 43 & 0 & 21 & 22 & $41.7 \%$ \\
TOTAL & 103 & $13.3(2.5)$ & 45 & 58 & 50 & 53 & $100 \%$
\end{tabular}

The special classes selected for this study were located in Adama, Asella, Bahir Dar and Hawassa. The special schools for the study were Mekane Yesus School for the Deaf in Hossana, Arba Minch Special School in Arba Minch, and Victory School for the Deaf in Addis Ababa (Table 2).

Table 2 Location and number of participants by the school setting

$\begin{array}{lcccccccr}\text { School setting } & \text { A/minch } & \text { Adama } & \text { Asella } & \text { Hawassa } & \text { B/Dar } & \text { Hossana } & \begin{array}{c}\text { AA } \\ \text { Victory }\end{array} & \text { Total } \\ \text { DHH in special class } & 0 & 10 & 5 & 9 & 5 & 0 & 0 & 29 \\ \text { DHH in special school } & 9 & 0 & 0 & 0 & 0 & 17 & 5 & 31 \\ \text { Hearing in regular school } & 1 & 12 & 10 & 8 & 12 & 0 & 0 & 43 \\ \text { Total } & 10 & 22 & 15 & 17 & 17 & 17 & 5 & 103\end{array}$




\section{Procedure}

Behavioural problems were measured with the Strengths and Difficulties Questionnaire (SDQ) (Goodman, 1997) using the self-report for children and adolescents SDQ(S) 11-17. Goodman's SDQ (Goodman, 1997) is a brief child behavioural screening questionnaire that asks about 25 negative and positive attributes, generating scores for five subscales: Conduct Problems, Hyperactivity-Inattention, Emotional Problems, Peer Problems and Prosocial Behaviour. The emotional and peer subscales could be categorized into an internalizing subscale and the behavioural and hyperactivity subscales into an externalizing subscale. There is also an impact supplement of the SDQ with the 25 items that asks whether the child thinks that she/he has a problem, and if so, enquire further about chronicity, distress, social impairment, and burden to others. The original SDQ measure for parents included a version translated into Amharic; however, a slight wording modification was made to the Amharic translation with respect to the self-report component. The adapted questionnaire was used to measure difficulties as perceived by the students themselves (emotional, conduct and peer problems; hyperactivity and prosocial behaviour). It contained 25 statements pertaining to the child (e.g., "many worries, often seems worried"; “constantly fidgeting or squirming"; "considerate of other people's feelings"); the children graded these statements as "not true", "somewhat true" or "certainly true". For the impact supplement questionnaire, children had to rate their behavioural or emotional difficulties in terms of interference in daily life as "not at all", "only a little", "quite a lot", or "a great deal".

The data were collected just before the end of the academic year in May - June, 2011. The 25 items and impact questions for SDQ measures were distributed to each child to fill out while the researcher presented them on an overhead projector. The researcher explained all the questions and instructions in sign language for DHH students and read them aloud for hearing students. They were 
presented in separate sessions for the hearing and DHH groups in order to avoid mixing sign and spoken languages. If the children did not understand a certain word, they were assisted with paraphrases. After replying to the 25 items, participants were asked to fill out the impact supplement questionnaire for the SDQ measures. At the beginning of the impact supplement, there was the following question: "Overall, do you think that you have difficulties in one or more of the following areas: emotions, concentration, behaviour or being able to get on with other people?" For this question, participants needed to reply as "No", "Yes-minor difficulties", "Yes-definite difficulties", or "Yes-severe difficulties". 30 participants (29\%) answered "No" to this particular question, and they did not continue with further testing. Among those who answered "No", the majority were hearing participants $32.6 \%$ (14/43). The remainder included 29\% (9/31) of DHH students in special schools and 24.1\% (7/29) of DHH students in special classes.

\section{Analysis}

All statistical analyses were performed using SPSS version 18.0. One-way ANOVA was used to compare differences in scores between the three school settings with a significance level set at $\mathrm{P} \leq$ .05. In estimating the magnitude or practical importance of the difference a rule of thumb suggested by Cohen (1988) for interpretation of eta $^{2}$ statistics was used. In One-way ANOVA eta ${ }^{2}$ values above .01 are considered as indicating small effect size, values above .06 a medium effect size and values above .14 a large effect size.

\section{Results}

The results show that the impact of socio-emotional problems is more severe across all dimensions for the DHH students; this finding holds true for those attending special classes and for those in special schools when compared to the hearing sample (Table 3). The DHH students in the two 
different settings differed significantly from the hearing children in all domains of socio-emotional problems, except in leisure activities and likelihood to become upset or distressed about difficulties.

Table 3 The impact of socio-emotional problems as perceived by the students $(\mathrm{N}=73)$

\begin{tabular}{|c|c|c|c|c|c|c|c|c|c|}
\hline \multirow[t]{2}{*}{ Impact items } & \multicolumn{2}{|c|}{$\begin{array}{l}\text { Hearing } \\
\text { students } \\
(\mathrm{N}=29)\end{array}$} & \multicolumn{2}{|c|}{$\begin{array}{l}\text { DHH in } \\
\text { special class } \\
(\mathrm{N}=22)\end{array}$} & \multicolumn{2}{|c|}{$\begin{array}{l}\mathrm{DHH} \\
\text { special } \\
\text { school } \\
(\mathrm{N}=22)\end{array}$} & \multirow[t]{2}{*}{$\mathrm{F}$} & \multirow[t]{2}{*}{ Sig. } & \multirow{2}{*}{$\begin{array}{l}\text { Eta- } \\
\text { squared } \\
\left(\eta^{2}\right)\end{array}$} \\
\hline & $\mathrm{M}$ & SD & $\mathrm{M}$ & SD & $\mathrm{M}$ & SD & & & \\
\hline How long difficulties being present & 1.97 & 1.05 & 2.73 & 1.20 & 3.05 & 0.99 & 6.78 & .002 & 0.16 \\
\hline Difficulties upset or distress me & 2.24 & 0.87 & 2.86 & 1.03 & 2.41 & 1.05 & 2.60 & .08 & 0.07 \\
\hline Difficulties interfere with home life & 1.83 & 1.00 & 2.86 & 0.99 & 2.23 & 0.87 & 4.94 & .01 & 0.12 \\
\hline Difficulties interfere with friendships & 1.83 & 0.89 & 2.45 & 1.10 & 2.45 & 0.96 & 3.59 & .033 & 0.09 \\
\hline Difficulties interfere with classroom learning & 1.72 & 0.88 & 2.76 & 1.09 & 2.64 & 1.18 & 7.66 & .001 & 0.18 \\
\hline Difficulties interfere with leisure activities & 1.62 & 0.90 & 2.18 & 1.14 & 2.00 & 0.98 & 2.11 & .129 & 0.06 \\
\hline $\begin{array}{l}\text { Difficulties make it harder for people around } \\
\text { me (family, friends, teachers, etc.) }\end{array}$ & 1.41 & 0.63 & 3.00 & 1.14 & 2.23 & \begin{tabular}{|l|}
0.87 \\
\end{tabular} & 20.2 & .000 & 0.37 \\
\hline SDQ Externalizing & 1.34 & 0.31 & 1.71 & 0.38 & 1.70 & \begin{tabular}{|l|}
0.34 \\
\end{tabular} & 14.19 & .000 & 0.221 \\
\hline
\end{tabular}

As measured by how long difficulties been present, DHH students seemed to have more chronic socio-emotional difficulties compared to hearing students $\left(\mathrm{P} \leq .002, \mathrm{~F}=6.78, \eta^{2}=.16\right)$, and the effect size of this difference was large $\left(\eta^{2}=.16\right)$. Emotional and behavioural problems interfere more in the home life of DHH students compared to their hearing peers $(\mathrm{P} \leq .01, \mathrm{~F}=4.94)$ and the difference had a medium effect size $\left(\eta^{2}=.12\right)$. Behavioural difficulties also interfere more in the classroom learning of DHH students than for their hearing peers $\left(\mathrm{P} \leq .001, \mathrm{~F}=7.66, \eta^{2}=.18\right)$, as indicated by the large effect size of the differences. There were also significant differences in relation to friendships between hearing students and the DHH group in both settings $(\mathrm{P} \leq .033, \mathrm{~F}=$ 3.59) with a medium effect size $\left(\eta^{2}=.09\right)$. However, hearing and DHH children differ most in the way behavioural and emotional difficulties interfere with getting along with people around them (P $<.001, \mathrm{~F}=20.2)$, as indicated by the massive effect size $\left(\eta^{2}=.37\right)$. 
A statistically significant difference also was found in the SDQ externalizing behavioural patterns of DHH students and hearing students. SDQ externalizing consists of the hyperactivity scale and conduct problems scale of the 25 SDQ measures. The DHH students in both the special schools and special classes scored higher results in the externalizing behavioural domains compared to hearing students, and the effect size of the differences was large $\left(\mathrm{P} \leq .000, \mathrm{~F}=14.19, \eta^{2}=.22\right)$.

Because there were differences with age across the three groups, we wanted to ascertain whether or not age affects the outcomes. Therefore, we added age as a co-variate to all of the equations with significant effect on the impact items. Adding age to the variable "difficulties interfere with home life" made it no longer significant. With all other variables, adding age as a covariate did not change the results in a meaningful way.

\section{Discussion}

This study investigated the impact of socio-emotional problems in DHH students compared to hearing students in Ethiopia. The data were collected through a survey administered to a sample of 103 grade four students attending a special school for the deaf, a special class for the deaf and a regular school. The survey instrument was the self-report measure of SDQ (Goodman, 1997) for children and adolescents. The comparison of the impacts of socio-emotional problems revealed significant differences between the DHH and hearing students, with severe impacts across most dimensions. The differences between the DHH students in the special class and special school settings were insignificant, and therefore, the presentation of results focused on the differences between DHH and hearing students. In this study, DHH students possessed significantly higher externalizing behaviour than their hearing counterparts. The pattern of these results support findings from earlier studies that have investigated the socio-emotional development and mental health of 
DHH children (Brown \& Cornes, 2014; Dammeyer, 2010; Fellinger et al., 2008; Hintermair, 2007; van Eldik et al., 2004; van Gent et al., 2007). This result was partially surprising as we expected better socio-emotional status among DHH students in the special schools compared to DHH students in the special classes. In the special schools where this data was collected there were better sign language skilled teachers, deaf adults who could be role models than in the special classes. In addition to this, the special schools have much more other DHH students, thus increasing the probability for creating better communication and social interaction. These conditions might result into lower socio-emotional problems for special school students, but this was not the case. The more restricted communication between DHH students and their families, hearing peers and the community seemed to affect negatively the socio-emotional development of all DHH students regardless of the educational settings. The emotional and behavioural problems seemed to interfere significantly in the home life of DHH children, in their interaction with people around them and in relation to friendships. This could be because of the communication difficulties from the early childhood and negative attitude in the society towards deafness. All of these DHH students come from hearing families in which the parents do not know sign language to communicate deeply and sensitively with their deaf children and this is likely to contribute to the findings. Interestingly the DHH students in both settings (special schools and special classes) reported that the socioemotional problems did not affect their leisure activities.

The findings of this study highlight the significance of early access to communication and language development, whether signed or spoken languages, which has been well documented also in previous research on the psychosocial development of DHH children (Calderon \& Greenberg, 2011) As Polat (2003) stated, the main obstacle deafness imposes is a communication problem, and the communicative development of the child starts at a very early age.

In Ethiopia, as in many other sub-Saharan African and low-income countries, society still holds negative attitudes and cultural beliefs characterizing disabilities, and affecting child's 
development and social participation (Derseh, 1995; Eide et al., 2011; Hartley, et al., 2005; Njelesani, et al., 2011; Parnes et al., 2009; Tirusew, 2005), which could contribute for the high score of socio-emotional problems in $\mathrm{DHH}$ children. Especially in rural areas, disability is perceived as a threat to the survival of the family (Eide et al., 2011; Njelesani et al., 2011). Deafness often is understood as demonic possession or as a punishment from God for the parent's sins. For this reason, parents often hide their deaf children from the public, especially in rural areas. A common view in society is to show pity to deaf children and to consider them as a burden because they are dependent and cannot be educated. Erroneous terms like "denkoro" and "duda" for hearing impairment in Amharic have a negative meaning. The terms imply that they are "idiots" who do not understand at all or can't be educated; such attitudes may directly or indirectly contribute to the DHH children's development of psychosocial problems. Usually parents in Ethiopia realize that their child can't hear when the child is at the age of three or above and fails to respond to sounds and communication. In sub-Saharan Africa, especially in rural areas where access to health care is limited, parents attempt various traditional practices that usually worsen temporary or mild hearing impairments and damage residual hearing (Olusanya, 2008). In Ethiopia, early diagnosis, screening tests and appropriate early intervention are not available, and, consequently, deaf children pass through these painful traditional practices and their precious early childhood without communication and language learning. By the time they come to school at 9-15 years of age, they often are beyond the age at which they could have learned fundamental basic skills more quickly and easily.

Among DHH people in Ethiopia, the students who participated in this study belong to the minority who attend school. Therefore, their wellbeing and learning require more attention. In four African countries, according to Eide et al. (2011), children with sensory impairments (hearing and visual impairments) are far more often excluded from primary education than children with other types of impairments, and $40-60 \%$ of adults with sensory impairments are illiterate. Due to the low 
gross enrolment rate (about 3\%) of children with disabilities in primary school (MoE, 2012), it can be assumed that the illiteracy rate for DHH adults is much higher in Ethiopia.

It is difficult to test behavioural and emotional problems of DHH children because there are no standard instruments available specifically for deaf children. Furthermore, as Cornes et al. (2006) have noted using standard written questionnaires underestimates the prevalence of psychological problems among deaf children and therefore sign language versions of instruments yields higher results. The translation of SDQ from another culture and from the English language to Amharic and then to Ethiopian Sign Language might have impacted DHH children's ability to understand, especially some concepts with negative expressions that were presented indirectly. Moreover, many DHH children, especially those who were attending special classes, had limited language skills (signed or spoken), which was addressed as much as possible by the assistance of the teachers and the researcher. These children usually started to learn Ethiopian Sign Language after they came to school. Therefore, it is important that future studies translate the SDQ self-report into Ethiopian Sign Language using a rigorous iterative process of translation and back-translation. The sample size in this study is small, consisting of only grade four students. In future studies, there should be larger sample sizes and longitudinal studies. This study, to our knowledge, is the first to investigate the behavioural and social problems of DHH children in sub-Saharan African countries.

In this study, DHH children expressed great concern about their socio-emotional difficulties. The high rates of socio-emotional problems in DHH children require joint attention by teachers, parents and professionals. In the conditions of Ethiopia, where access to other services and support is limited, teachers need training in how to overcome the obstacles faced by these children. Improvements in socio-emotional development enable DHH students to learn, complete their education and become productive citizens. Intervention programs need to be developed for the children, parents, and teachers. Such programs would provide knowledge about deafness, communication and languages, coping strategies and psychosocial support. Prior to attending 
school, DHH students' psychosocial development is affected by parental child-rearing attitudes and community-child relationships. Therefore, the early years are crucial in optimal development of the DHH child. In accordance with Muderedzi and Ingstrad (2011), a culturally-appropriate approach to disability, including deafness and hearing impairments, requires the involvement of parents and families. Parents of DHH children need to learn sign language in order to provide a rich and fluent sign language environment for the child from an early age. Enhancing the interaction of DHH children with their environment will help improve socio-emotional and self-concept development. 


\section{References}

Ayana, A. and Lehtomäki, E. 2006 'Including special needs education services in the federal level education sector development program in Ethiopia: Process, strategic priorities and implications', in H. Savolainen, M. Matero and H. Kokkala (eds), When Education for All Means All: Experiences in Three African Countries with EFA and Children with Disabilities, pp. 69-83, Ministry for Foreign Affairs, Finland.

Bat-Chava, Y. 2000 'Diversity of Deaf Identities', American Annals of the Deaf, 145 (5): 420-428.

Brown, P. M. And Cornes, A. J. 2014 'Mental health of Deaf and Hard of Hearing Adolescents: What the students say', Journal of Deaf Studies and Deaf Education, doi: 1093/deafed/enu/031.

Calderon, R., and Greenberg, M. 2011 'Social and emotional development of deaf children: Family, school, and program effects', in M. Marschark and P. E. Spencer (eds.), Oxford Handbook of Deaf Studies, Language and Education ( $2^{\text {nd }}$ ed., Vol. 1), pp. 188-199. New York: Oxford University Press.

Cartledge, G., Paul, P. V., Jackson, D., and Cochran, L. L. 1991 'Teachers' perceptions of the social skills of adolescents with hearing impairment in residential and public school settings', Remedial and Special Education, 12: 34-39.

Cohen, J. 1988 Statistical power analysis for the behavioural sciences (2nd ed.). Hillsdale, NJ: Lawrence Erlbaum Associates.Cornes, A. J., Rohan, M. J., Napier, J. and Rey, J. M. 2006 'Reading the signs: impact of signed versus written questionnaires on the prevalence of psychopathology among deaf adolescents', Australian and New Zealand Journal of Psychiatry, 40(8): $665-673$.

Dammeyer, J. 2010 'Psychosocial development in a Danish population of children with cochlear implants and deaf and hard-of-hearing children', Journal of Deaf Studies and Deaf Education, 15: 50-58. doi:10.1093/deafed/enp024. 
Derseh, T. B. 1995 Meanings attached to disability, attitudes towards disabled people, and attitudes towards integration, $\mathrm{PhD}$ diss., Jyväskylä Studies in Education, Psychology and Social Research no. 118: University of Jyväskylä.

Eide, A. H., Loeb, M. E., Nhiwatiwa, S., Munthali, A., Ngulube, T. J. and van Rooy, G. 2011 'Living conditions among people with disabilities in developing countries', in A. H. Eide and B. Ingstad. (eds) Disability and Poverty: A Global Challenge, pp. 55-70. Bristol: The Policy Press. van Eldik, T., Treffers, P. D., Veerman, J. W., and Verhulst, F. C. 2004 'Mental health problems of deaf children as indicated by parents' response to the child behaviour checklist', American Annals of the Deaf, 148: 390-395.

Fellinger, J., Holzinger, D., Sattel, H., and Laucht, M. 2008 'Mental health and quality of life in deaf pupils', European Child \& Adolescent Psychiatry, 17: 414-423. doi:10.1007/s00787-0080683-y.

van Gent, T., Goedhart, A. W., Hindley, P. A., and Treffers, P. D. A. 2007 'Prevalence and correlates of psychopathology in a sample of deaf adolescents', Journal of Child Psychology and Psychiatry, 48: 950-958.

Goedele, C. 2011 'Fostering deaf people's empowerment: the Cameroonian deaf community and epistemological equity', Third World Quarterly, 32(8): 1419-1435.

Goodman, R. 1997 'The strengths and difficulties questionnaire: A research note', Journal of Child Psychology and Psychiatry, 38: 581-586. doi:10.1111/j.1469-7610.1997. tb01545.x.

Hartley, S., Ojwang, P, Baguwemu, A., Ddamulira, M. and Chavut, A. 2005 'How do carers of disabled children cope? The Ugandan perspective', Child: Care, Health and Development 31(2): $167-80$.

Hindley, P., Hill, P. D., McGuigan, S., \& Kitson, N. 1994 'Psychiatric disorder in deaf and hearing impaired children and young people: A prevalence study', Journal of Child Psychology and Psychiatry, 35: 917-934. 
Hintermair, M. 2007 'Prevalence of socio-emotional problems in deaf and hard of hearing children in Germany, American Annals of the Deaf, 152: 320-330.

Hintermair, M. 2013 'Executive functions and behavioural problems in deaf and hard-of-hearing students at general and special schools', Journal of Deaf Studies and Deaf Education, 18(3): 344-359. doi:10.1093/deafed/ent003.

Kauffman, J. M., and Landrum, T. J. 2013Characteristics of Emotional and Behavioural Disorders of Children and Youth (10th ed.). Boston: Pearson/Merrill.

Keilmann, A., Limberger, A., and Mann, W. J. 2007 'Psychological and physical well-being in hearing-impaired children', International Journal of Pediatric Otorhinolaryngology, 71: 17471752.

Kiyaga, N. B. and Moores, D. F. 2003 'Deafness in sub-Saharan Africa', American Annals of the Deaf, 148(1): pp. $18-24$.

Marschark, M. 1993 Psychological Development of Deaf Children. London: Oxford University Press.

Marschark, M., and Knoors, H. 2012 'Educating deaf children: Language, cognition, and learning', Deafness \& Education International, 14(3): 136-160.

Marschark, M., and Wauters, L. 2011 'Cognitive functioning in deaf adults and children', in M. Marschark and P. E. Spencer (eds), Oxford handbook of deaf studies, language, and education ( $2^{\text {nd }}$ ed., Vol. 1) pp. 486-499, New York: Oxford University Press.

Mayberry, R. I. 2003 'Cognitive development in deaf children: The interface of language and perception in neuropsychology', in S. J. Sagalowitz and I. Rapin (eds) Handbook of Neuropsychology, (2 ${ }^{\text {nd }}$ ed., Vol. 8, Part II) pp. 487-523. Amsterdam: Elsevier.

Meadow, K. P. 2005 'Early manual communication in relation to the deaf child's intellectual, social, and communicative functioning', Journal of Deaf Studies and Deaf Education, 10: 321329. 
MoE. 2012 Annual report of the Ethiopian Ministry of Education. Addis Ababa, Ethiopia.

Muderedzi, J. and Ingstad, B. 2011 'Disability and social suffering in Zimbabwe', in A.H. Eide and B. Ingstad. (eds) Disability and Poverty: A Global Challenge, pp. 171-188. Bristol: The Policy Press.

Mulat, M. 2011 Annual report of SEP deaf. Addis Ababa, Ethiopia.

Njelesani, J., Couto, S. and Cameron, D. 2011 'Disability and rehabilitation in Tanzania: A review of the literature', Disability and Rehabilitation, 33(23-24): 2196-2207.

Olusanya, B. O. 2008 'Priorities for early hearing detection and intervention in sub-Saharan Africa', International Journal of Audiology, 47 (1): S3-S13.

Parnes, P., D. Cameron, N. Christie, L. Cockburn, G. Hashemi, and K. Yoshida. 2009 'Disability in low-income countries: Issues and implications', Disability and Rehabilitation 31(14): 11701180.

Polat, F. 2003 'Factors affecting psychosocial adjustment of deaf students', Journal of Deaf Studies and Deaf Education, 8: 325-339.

Preisler, G. 1999 'The development of communication and language in deaf and severely hard of hearing children: Implications for the future', International Journal of Pediatric torhinolaryngology, 49(1): S39-S43.

Sinkkonen, J. 1994 Hearing impairment, communication and personality development. Helsinki, Finland: University of Helsinki, Department of Child Psychiatry.

Stacey, P.C., Fortnum, H.M., Barton, G.R., and Summerfield, A.Q. 2006 'Hearing-impaired children in the United Kingdom: Auditory performance, communication skills, educational achievements, quality of life, and cochlear implantation', Ear and Hearing, 27: 161-186.

Theunissen, S.C. et al. 2014 'Behavioural problems in school-aged hearing-impaired children: The influence of socio-demographic, linguistic, and medical factors', European Child \& Adolescent Psychiatry, 23 (4): 187-196. DOI: 10.1007/s00787-013-0444-4. 
Tirussew, T. 2005 Disability in Ethiopia: Issues, Insights and Implications. Addis Ababa: Addis Ababa University Printing Press.

Tucchi, D. L., Merson, M. H. and Wilson, B. S 2009 'A summary of the literature on global hearing impairment: Current status and priorities for action', Otology \& Neurotology, 31: 31-41.

WHO 2013 'Deafness and hearing loss', viewed July 11, 2013,

http://www.who.int/mediacentre/factsheets/fs300/en/. 